\title{
Diet Control Diet to Achieve Euglycemia Induces Tau Hyperphosphorylation Via AMPK Activation in the Hippocampus of Diabetic Rats
}

\author{
Jun-Ho Lee ${ }^{1,2,7}$, Yun-Hee Noh ${ }^{1, \#,}$, Mingli Jin ${ }^{5}$, Jin-Seong Kim ${ }^{6}$, Sang-Don Han ${ }^{3,4, *}$ \\ 1 Department of Biochemistry, Konkuk University School of Medicine, Seoul 05029, Republic of Korea \\ 2 Department of Biomedical Chemistry, Konkuk University College of Biomedical and Health Science, Chungju 27478, \\ Republic of Korea \\ 3 Department of Medical Education, Konkuk University School of Medicine, Seoul 05029, Republic of Korea \\ 4 Department of Neurology, Konkuk University School of Medicine, Chungju Hospital, Chungju 27376, Republic of \\ Korea \\ 5 Gachon Institute of Pharmaceutical Sciences, College of Pharmacy, Gachon University 21936, Republic of Korea \\ 6 International Ginseng \& Herb Research Institute, Chungnam, 32724, Republic of Korea \\ * Correspondence: Department of Medical Education, Konkuk University School of Medicine, 120 \\ Neungdong-ro, Gwangjin-gu, Seoul 05029, Republic of Korea. Email: hihanol@kku.ac.kr Tel: +82-10-4701- \\ 7448
}

\begin{abstract}
Alzheimer's disease (AD) is a chronic neurodegenerative disease, and typical pathologic findings include abnormally hyperphosphorylated tau aggregation and neurofibrillary tangles. Insulin resistance and hyperglycemia have been assessed as risk factors for AD development. As the maintenance of optimal blood glucose levels is an important indicator of diabetes mellitus (DM) treatment, diet control is essential. AMPK is a crucial sensor of cellular bioenergetics for controlling anabolic and catabolic metabolism. Diet restriction to achieve euglycemia can increase AMPK activity in the liver and heart. Since AMPK is a direct regulator of tau phosphorylation, we hypothesized that strict diet control to achieve euglycemia affects tau protein phosphorylation through increased AMPK activity in the hippocampus of DM rats. To confirm this hypothesis, we generated insulin-deficient DM rats by subtotal pancreatectomy. Animals were categorized into the restriction (R) group, control (C) group and ad libitum (AL) group according to the diet. We found that tau phosphorylation was significantly increased in the $\mathrm{R}$ group compared with the $\mathrm{C}$ or $\mathrm{AL}$ group. AMPK activity in the $\mathrm{R}$ group significantly increased compared to that of the $\mathrm{C}$ group or AL group, as expected. Furthermore, the $\mathrm{R}$ group showed more critical tau pathology in the hippocampus than the other groups. These results suggest that diet control to achieve euglycemia in insulin-deficient DM conditions is harmful because of the increased possibility of $\mathrm{AD}$ development through increased tau phosphorylation by AMPK activation in the hippocampus. We propose that not only hyperglycemia but also euglycemia, which is beneficial in DM patients, must be considered a potential risk factor for AD development, especially when euglycemia is achieved by diet control during insulin deficiency.
\end{abstract}

Keywords: Alzheimer's disease, Diabetes, Diet control diet, AMPK, Tau hyperphosphorylation

\section{Introduction}

Alzheimer's disease (AD) is characterized by progressive neuronal loss and synaptic injury. The primary pathologic hallmarks of $\mathrm{AD}$ are neurofibrillary tangles (NFTs) and amyloid beta (A $\beta$ ) plaques. NFTs are formed by intracellular aggregation of paired helical filaments, which are composed of a microtubule-associated protein known as tau that shows abnormal hyperphosphorylation [1]. A $\beta$ plaques are extraneuronal deposits of $A \beta$ that develop through amyloid precursor protein cleavage by beta secretase and gamma secretase [2]. Although much 
attention has recently been focused on $\mathrm{A} \beta$ as the causative agent in AD, NFT pathology correlates better with cognitive decline in AD than amyloid pathology [3].

TAccumulating evidence suggests that diabetes mellitus (DM) is associated with the development of $\mathrm{AD}$. An epidemiologic study demonstrated that $\mathrm{DM}$ patients have a high risk of developing $\mathrm{AD}$, which is independent of the risk of vascular dementia [4]. Moreover, patients with $\mathrm{DM}$ had a nearly 2-fold increased incident risk of $\mathrm{AD}[5]$ and a $65 \%$ increase in the risk of developing AD compared with those without DM [6]. Many experimental animal models of DM have also shown AD pathology, including tau hyperphosphorylation $[7,8]$.

$\mathrm{DM}$ is characterized by hyperglycemia due to the combination of insulin resistance and impaired insulin secretion. The goal of DM treatment has been correction of hyperglycemia to euglycemia or near-euglycemia because hyperglycemia is associated with the development of DM complications and mortality $[9,10]$. Thus, diet control is highly emphasized as a method of good glycemic control to achieve and maintain euglycemia in patients with DM [11,12].

Our previous study demonstrated that diet control to achieve euglycemia reduces the weight of the heart, liver, skeletal muscle, and epididymal fat mass as well as body weight in insulin-deficient DM rats via excessive autophagy [13]. Following this study, we also revealed that diet control to achieve euglycemia is deleterious in the insulin-deficient state due to increased apoptosis and autophagy in the liver via AMPK activation [14].

Meanwhile, recent studies have demonstrated that AMPK plays a role in tau kinase in AD development, in which AMPK itself phosphorylates tau at Ser262 and Ser396 by altering the microtubule binding of tau [15]. AMPK is also activated abnormally in tangle- and pre-tangle-bearing neurons in AD and multi-tau phosphorylation sites [16]. Therefore, we hypothesized that AMPK activated by diet control to achieve euglycemia may influence the development of $\mathrm{AD}$ in the brains of insulin-deficient DM animals through tau hyperphosphorylation in the brain and the loss of brain weight, as the loss of brain volume in various regions of the brain is a strong indicator of $\mathrm{AD}$ pathophysiology [17].

To confirm our hypothesis, we generated insulin-deficient DM rats by subtotal pancreatectomy to mimic end-stage type $2 \mathrm{DM}$ and induced euglycemia in the DM rats by diet control, where the DM rats were fed isocalories similar to the sham control rats. Then, we compared the body and the brain weights and tau phosphorylation in the experimental groups to determine whether diet control to achieve euglycemia in insulin-deficient DM affects the development of NFTs though activated AMPK. In addition, we also examined the phosphorylation level of the Akt-GSK-3 $\beta$ axis, a typical pathway for the development of NFT that is regulated by insulin and protein phosphatase 2A (PP2A). PP2A is known to regulate AMPK activation and tau phosphorylation [18,19].

\section{Results}

\subsection{Control of the food intake rate during the entire study}

Figure 1A shows changes in the rate of food intake ( $\mathrm{g} / \mathrm{kg}$ of body weight/day) of all groups throughout the study. The rate of food intake in the $C$ group continuously decreased during the entire study; however, the rate of food intake in the Px groups increased significantly during induction of diabetes compared with that of the C group. The rate of food intake in the AL group was continuously increased for 4 weeks after surgery and then maintained until the end of the study. During the diet control period, the rate of food intake in the $\mathrm{R}$ group was decreased as in the $\mathrm{C}$ group. In addition, the food intake rates of the $C$ and $R$ groups were $1 / 3$ of that of the AL group and were not significantly different during the diet control period. 

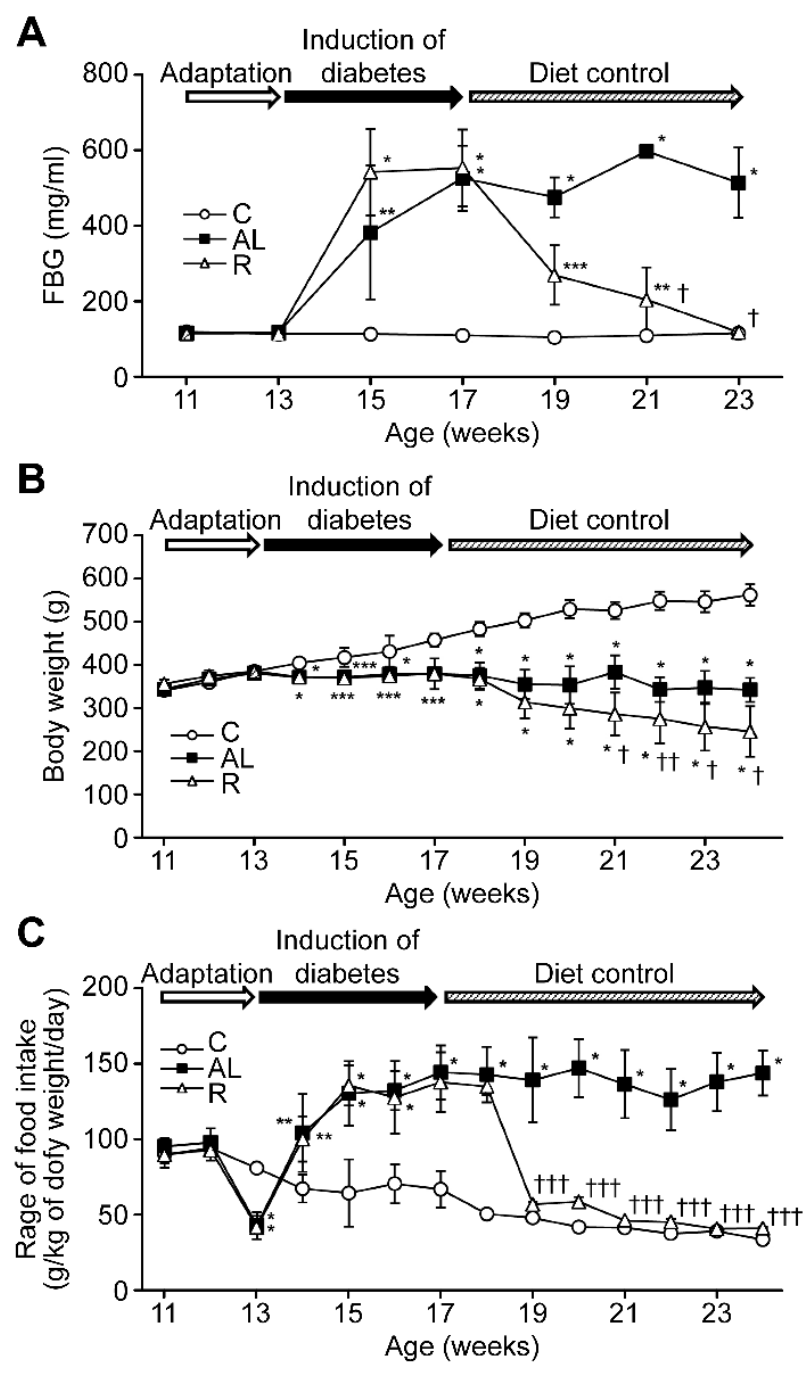

Figure 1. Sequential changes in (A) fasting blood glucose (FBG) levels, (B) body weights, and (C) daily food intake (g per kg body weight per day). Data are presented as means \pm SD and were analyzed with one-way ANOVA followed by Tukey's post hoc test. C: sham-operated rats fed ad libitum; AL: pancreatectomized diabetic rats fed ad libitum; R: pancreatectomized diabetic rats fed diet control to achieve euglycemia during the diet control period. $* P<0.001, * * P<0.05$, and $* * * P<$ 0.01 versus $C ;+P<0.01,++P<0.05$, and $+++P<0.001$ versus AL.

\subsection{Changes in fasting blood glucose levels ( $m g / d l)$ during the entire study}

Figure 1B shows changes in the fasting blood glucose (FBG) levels of all groups throughout the study. The FBG levels of the $C$ group maintained euglycemia throughout the study. However, the FBG levels of the Px groups were increased significantly and showed hyperglycemia during the induction of the diabetes period compared with those of the $C$ group (approximately 4.2-fold). During the diet control period, the FBG levels of the R group decreased similar to those in the C group, while the AL group maintained hyperglycemia until the end of the study.

\subsection{Changes in body weight $(g)$ during the entire study}

Figure 1C shows changes in the body weight ( $\mathrm{g}$ ) of all groups throughout the study. The body weight of the $C$ group increased continuously throughout the study; however, the body weight of the AL group was maintained after surgery until the end of the study. The body weight of the $\mathrm{R}$ group was maintained during the induction of diabetes but decreased continuously and significantly during the diet control period compared with that of the AL group. 
To confirm that we generated insulin deficiency in the Px groups, we measured serum insulin and plasma C-peptide levels. Figure 2 shows the mean plasma insulin (A) and plasma C-peptide (B) levels. The mean serum insulin levels of the Px groups, including the AL group and $\mathrm{R}$ group, were $7.9 \%$ and $4.4 \%$ of that of the $C$ group, respectively [C, $77.2 \pm 61.5$ vs AL, $6.1 \pm 2.9(P=0.011)$ or $R, 3.4 \pm$ $1.3(P=0.012)]$. The mean plasma $C$-peptide levels in the Px groups, including the AL group and $\mathrm{R}$ group, were $19.1 \%$ and $8.7 \%$ of that of the $C$ group, respectively $(C, 1.472 \pm 0.389$ vs AL, $0.281 \pm 0.185$ or R, $0.128 \pm 0.135 ; P<0.001$ for all).

A
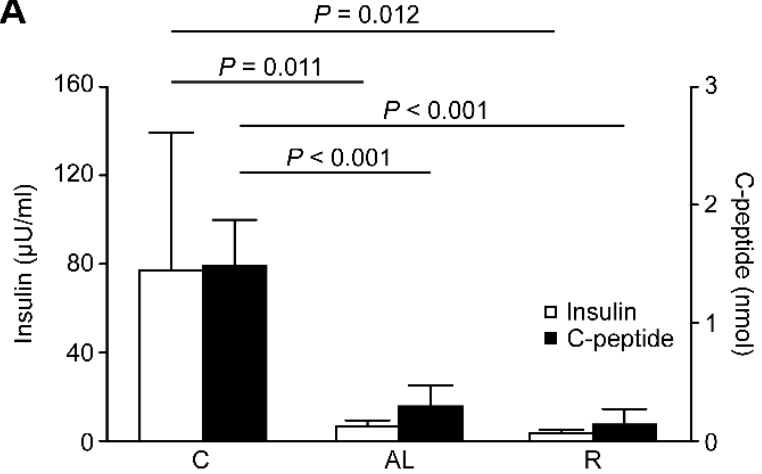

B

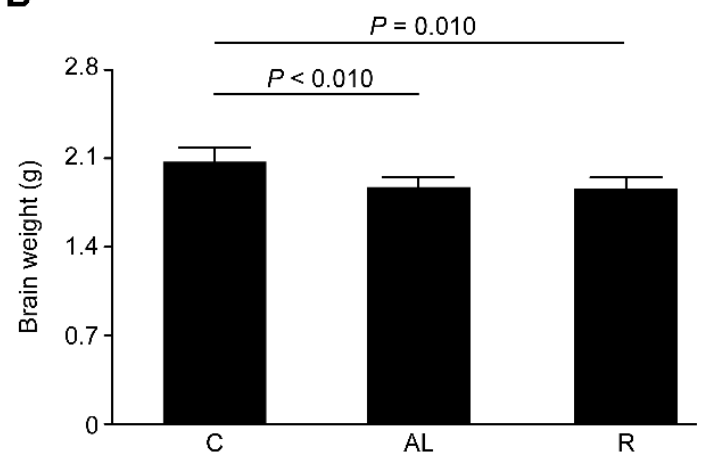

Figure 2. Endogenous plasma insulin and C-peptide levels. Data are presented as means \pm SD and were analyzed with one-way ANOVA followed by Tukey's post hoc test. C: sham-operated rats fed ad libitum; AL: pancreatectomized diabetic rats fed ad libitum; R: pancreatectomized diabetic rats fed diet control to achieve euglycemia during the diet control period.

\subsection{Induction of AMPK activity and tau hyperphosphorylation in the hippocampus}

To confirm that the diet control to achieve euglycemia induces AMPK activity and tau hyperphosphorylation in the hippocampus of insulin-deficient DM rats, we investigated the phosphorylation of AMPK and tau phosphorylated at Ser ${ }^{199 / 202}$ and Ser ${ }^{396}$ (Figure 3). AMPK phosphorylation in the $\mathrm{R}$ group was significantly increased approximately 3.1-fold compared to that of the $C$ group $(P<0.001)$, and AMPK phosphorylation was also increased approximately 2.5 -fold compared to that of the AL group $(P<0.001)$. However, there was no significant difference in AMPK phosphorylation between the C and AL groups. Tau phosphorylation at Ser ${ }^{199 / 202}$ of the Px groups (the AL and R groups) was significantly increased approximately 2.4- and 5.0-fold, respectively, compared to that of the $C$ group ( $C$ vs AL, $P=0.036$ or $R, P<0.001$ ). Furthermore, the level in the $\mathrm{R}$ group significantly increased by approximately 2.1-fold of that of AL (AL vs $\mathrm{R}, P=0.002$ ). Tau phosphorylation at Ser ${ }^{396}$ of the Px groups also significantly increased by approximately 2.2- and 3.1fold, respectively, compared to that of the $C$ group ( $\mathrm{C}$ vs $\mathrm{AL}, P=0.018$ or $\mathrm{R}, P=0.001$ ). Furthermore, the level in the R group significantly increased by approximately 1.4-fold of that in the AL group (AL vs $\mathrm{R}, P=0.047)$. 
A

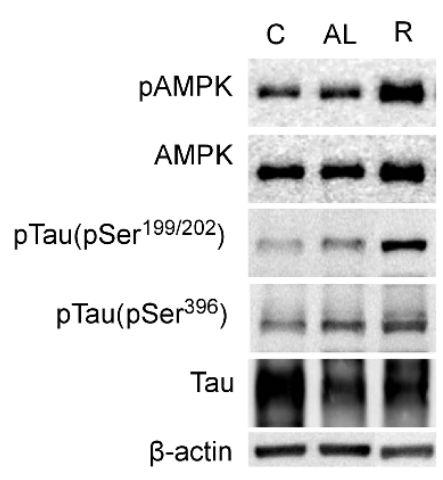

C

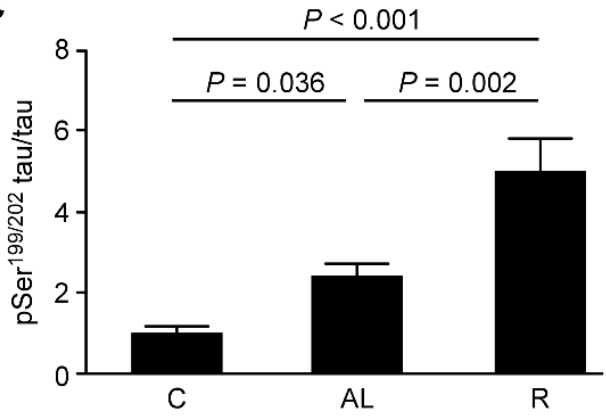

B

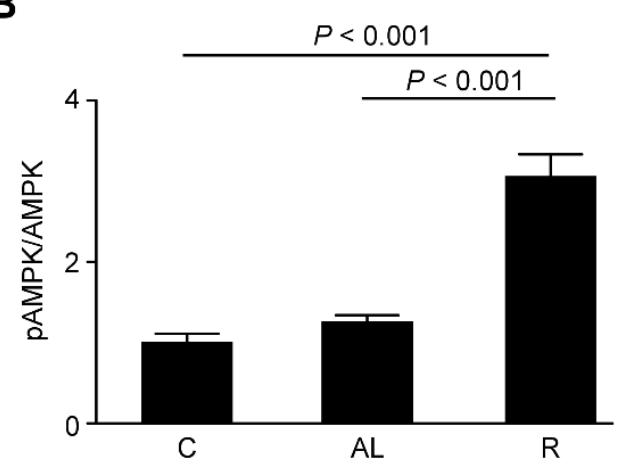

D

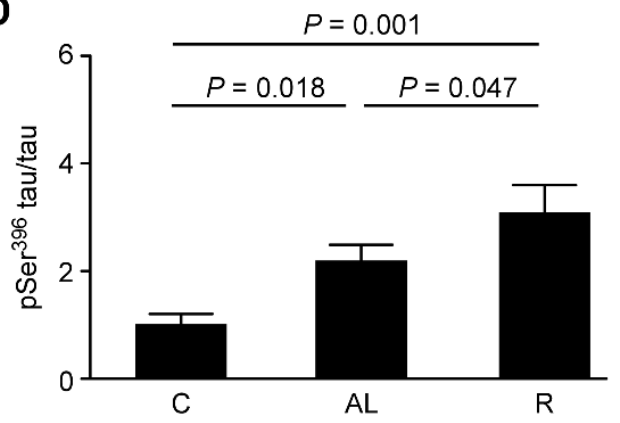

Figure 3. (A) Western blot analyses. (B) The ratio of pAMPK to AMPK. The ratio of (C) ptau at Ser199/202, and (D) ptau at Ser396 to tau. The blots shown are representative of triplicates. Data are presented as means \pm SD and were analyzed with one-way ANOVA followed by Tukey's post hoc test. C: sham-operated rats fed ad libitum; AL: pancreatectomized diabetic rats fed ad libitum; R: pancreatectomized diabetic rats fed diet control to achieve euglycemia during the diet control period.

\subsection{Akt, GSK-3 $\beta$, and PP2A activities in the hippocampus}

To determine the mechanism for tau phosphorylation in the hippocampus of euglycemic insulin-deficient DM rats, we examined the Akt and GSK-3 $\beta$ phosphorylation levels (Figure 4). Akt phosphorylation in the AL group significantly increased by approximately 2 -fold of that of the $C$ group ( $\mathrm{AL}$ vs $\mathrm{C}, P=0.003$ or $\mathrm{R}, P=0.002$ ). However, there was no significant difference between the $\mathrm{C}$ and $\mathrm{R}$ groups. GSK-3 $\beta$ phosphorylation in the $\mathrm{AL}$ and $\mathrm{R}$ groups significantly increased by approximately 2.6- and 2.0-fold, respectively, compared to that of the $\mathrm{C}$ group ( $\mathrm{C}$ vs $\mathrm{AL}, P<0.001$ or $\mathrm{R}, P=0.002)$. However, the level of the $\mathrm{R}$ group significantly decreased by approximately $76.4 \%$ of that of the AL group (AL vs R, $P=0.016$ ).

To further explain the mechanism of AMPK activation and tau phosphorylation in the hippocampus of euglycemic insulin-deficient DM rats, we investigated PP2A activity (Fig. 4). The PP2A phosphorylation of Px groups significantly decreased by approximately $64 \%$ compared to that of the $\mathrm{C}$ group ( $\mathrm{C}$ vs AL or $\mathrm{R}, P=0.021$ for all). However, there was no significant difference between the Px groups. 
A

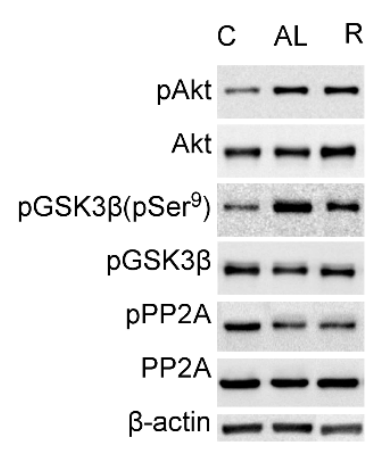

C

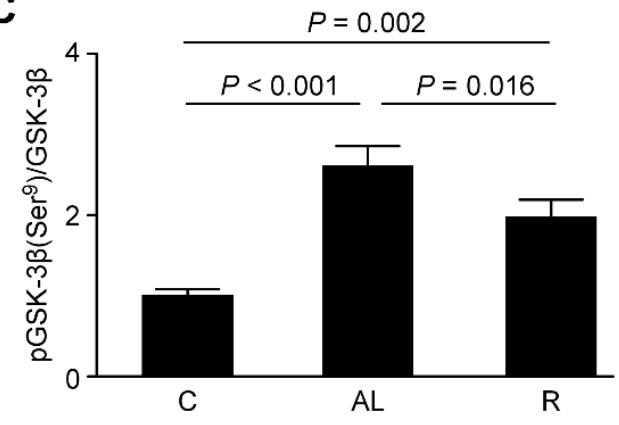

B

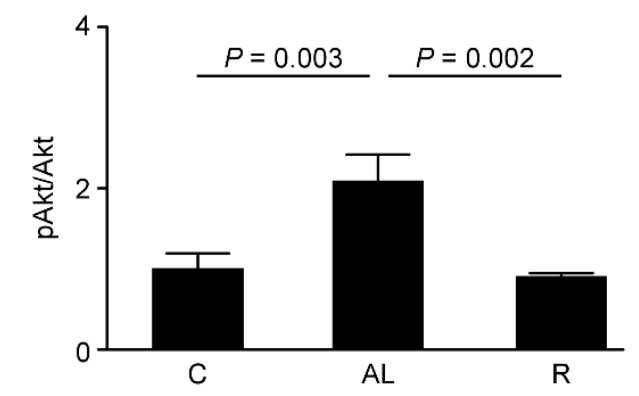

D

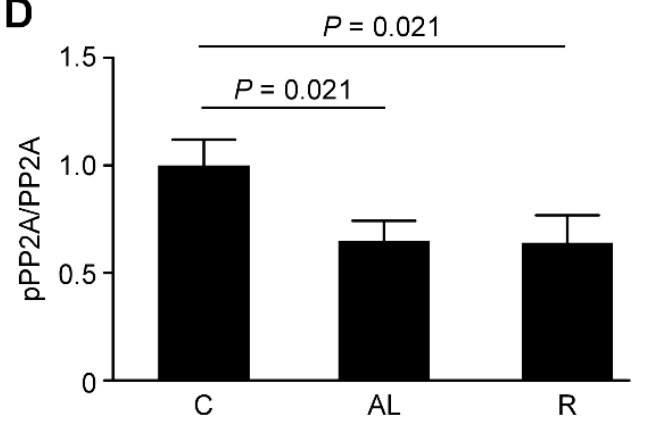

Figure 4. (A) Western blot analyses. (B) The ratio of pAkt to Akt. (C) The ratio of pGSK-3 $\beta$ at Ser9 to GSK-3 $\beta$. (D) The ratio of pPP2A to PP2A. The blots shown are representative of triplicates. Data are presented as means \pm SD and were analyzed with one-way ANOVA followed by Tukey's post hoc test. C: sham-operated rats fed ad libitum; AL: pancreatectomized diabetic rats fed ad libitum; R: pancreatectomized diabetic rats fed diet control to achieve euglycemia during the diet control period.

\subsection{Histopathology of the hippocampal CA1 region}

To determine whether diet control to achieve euglycemia induces tau hyperphosphorylation, we investigated the AD pathology in the CA1 region of the hippocampus (Figure 5). CV staining showed that the cell layer of the AL group was thinner than that of the $\mathrm{C}$ group, but the cell layer of the R group was thinnest in all groups. Furthermore, IHC staining showed that tau aggregation increased in the AL group compared to the $\mathrm{C}$ group, but it was highest in the R group.
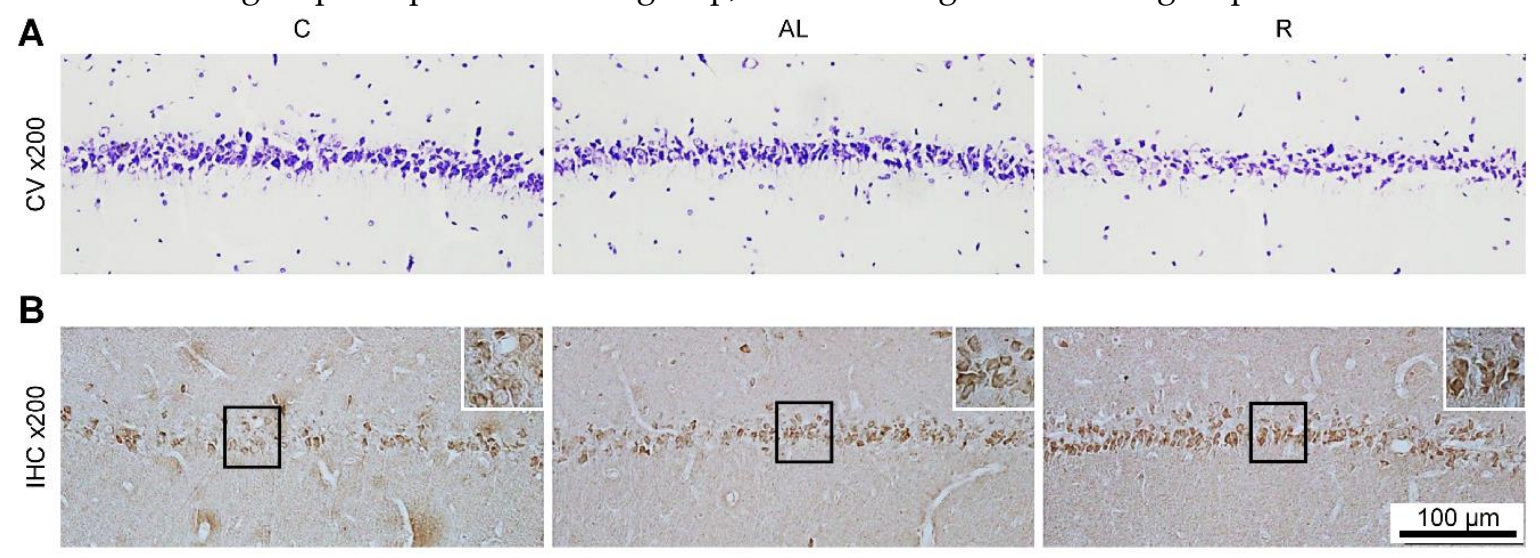

Figure 5. Hippocampus CA1 region histology. (A) Crystal violet staining. (B)

Immunohistochemistry of Tau. C: sham-operated rats fed ad libitum; AL: pancreatectomized diabetic rats fed ad libitum; R: pancreatectomized diabetic rats fed diet control to achieve euglycemia during the diet control period. 


\section{Discussion}

In this study, we demonstrated that a diet control diet to achieve euglycemia induced AMPK activation and tau hyperphosphorylation in the hippocampus of insulin-deficient DM rats. We suggest that diet control to achieve euglycemia has a dangerous effect on maintaining energy metabolism and structural function, although FBG levels obtained euglycemia in the hippocampus of insulin-deficient DM animals.

AMPK is a cellular sensor that regulates the activity of various metabolic enzymes to maintain energy homeostasis. AMPK is a heterotrimeric Ser/Thr protein kinase composed of catalytic $\alpha$ subunit and two regulatory $\beta$ and $\gamma$ subunits. This kinase is activated via Thr ${ }^{172}$ phosphorylation in the activation loop of the catalytic $\alpha 2$ subunit during energy stress by different upstream kinases [20]. AMPK is expressed in most mammalian tissues and cell types, including cerebral neurons, and is thought to play an important role in regulating energy homeostasis [21].

In this study, we found that AMPK activation was highly increased in the R group compared with the other groups, although FBG did not differ from that of the $C$ group. We also confirmed that phospho Ser ${ }^{199 / 202}$ and Ser $^{396}$ tau proteins were highly increased according to increased AMPK activity in the hippocampus of insulin-deficient DM rats fed a diet control diet to achieve euglycemia compared with those of hyperglycemic DM rats fed ad libitum (Figure 3). Furthermore, tau phosphorylation was increased in hyperglycemic DM rats compared with the $C$ group rats, but it was dramatically increased in the R group, in which AMPK was increased, compared with the AL group. Recently, many studies have reported that AMPK plays a key role in tau kinase and directly regulates various tau phosphorylation sites $[16,22,23]$. We suggest that the diet control to achieve euglycemia may aggravate $A D$ as increased AMPK activity leads to tau hyperphosphorylation in the hippocampus of insulin-deficient DM animals.

As Akt-GSK3 $\beta$ is known to be a typical upstream pathway involved in the regulation of tauopathy in the development of $\mathrm{AD}$, we also investigated whether diet control to achieve euglycemia affects Akt-GSK3 $\beta$ signaling. In this study, we confirmed that phosphorylation of Akt and GSK3 $\beta$ Ser ${ }^{9}$ in the AL group, which was maintained with hyperglycemia by ad libitum feeding in insulin-deficient conditions, was significantly increased compared to that in the $\mathrm{C}$ group. However, the phosphorylation of GSK3 $\beta$ Ser ${ }^{9}$ in the R group, which was fed the diet control diet to achieve euglycemia in insulin-deficient conditions, was still significantly increased compared to that of the $C$ group, although the phosphorylation of Akt did not differ compared to that of the $C$ group (Figure 4). GSK3 $\beta$ is phosphorylated at Ser ${ }^{9}$ by activated Akt, which is regulated by hyperglycemia in the brain [24], and this study could explain the Akt-GSK3 $\beta$ activity in the hippocampus of hyperglycemic $\mathrm{DM}$ rats fed ad libitum. However, it is unclear whether Akt-GSK3 $\beta$ activity in the hippocampus of insulin-deficient DM rats fed a diet control diet achieves euglycemia. To explain this, we investigated the activity of PP2A, which is a kinase inhibitor.

PP2A is mainly a soluble protein in the cytoplasm, but it is also found in the nucleus, mitochondria, cytoskeleton and cell membrane. PP2A has multiple roles in cell cycle regulation, cell morphology and signal transduction by dephosphorylating different substrates and critically regulating the integrity of the cytoskeleton [25]. PP2A is also known to directly dephosphorylate various serine sites of tau, including Ser ${ }^{199 / 202}$ and Ser ${ }^{396}[26]$, and regulates the activation of GSK3 $\beta$ via dephosphorylation at Ser ${ }^{9}$ [27]. In addition, PP2A activity was decreased, and decreased PP2A phosphorylation of GSK3 $\beta$ at Ser ${ }^{9}$ in the cortex and hippocampus of streptozotocin-injected DM mice was observed compared with that in normal mice [28]. Our study showed that PP2A activity was decreased in the hippocampus of Px rats compared with the $C$ group rats. Therefore, we speculate that the increase in phosphorylation of GSK3 $\beta$ Ser ${ }^{9}$ can be explained by PP2A, which was dephosphorylated, despite the decrease in Akt phosphorylation in the R group.

PP2A is also known to directly phosphorylate various serine sites of tau, including Ser ${ }^{199 / 202}$ and $\operatorname{Ser}^{396}$ [26], and regulate the phosphorylation of the AMPK $\alpha$-subunit at Thr ${ }^{172}$ [18]. Although the exact pathway between AMPK and PP2A was unclear in this study, we speculate that insulin-deficient DM conditions and diet control to achieve euglycemia dephosphorylate PP2A and affect the 
phosphorylation of AMPK and GSK3 $\beta$; therefore, this protein seems to be involved in tau hyperphosphorylation.

Based on these findings, we suggest that GSK3 $\beta$ activity is not accompanied by tau hyperphosphorylation in the hippocampus of insulin-deficient DM rats as PP2A is decreased. However, as a diet control diet to achieve euglycemia has a starvation-like effect, this diet induces tau hyperphosphorylation via activated AMPK and decreases PP2A in the hippocampus of insulindeficient DM rats compared with that of hyperglycemic DM rats fed ad libitum. The biguanide metformin has been reported to have a beneficial effect on tau hyperphosphorylation via activated AMPK and PP2A in vivo and in vitro [29]. However, our study showed that increased AMPK has a harmful effect on tau hyperphosphorylation in the hippocampus in insulin-deficient DM conditions, such as the last stage of T2DM, because the in vivo and in vitro models used in this study were not under DM-related tauopathy. Figure 6 briefly shows the protein signal transduction that occurs in the insulin-deficient hippocampus suggested in this study.

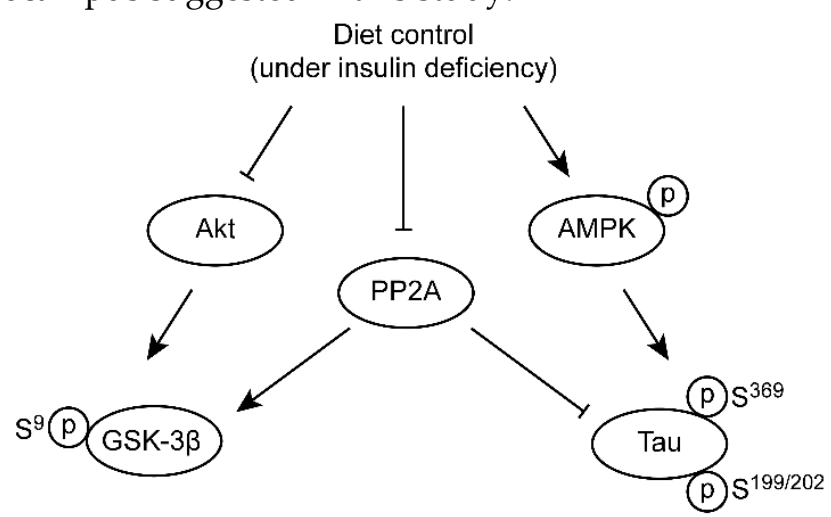

Figure 6. Signal transduction that concerned the tau hyperphosphorylation in the brain under insulin deficiency condition.

Therefore, we suggest that diet control to achieve euglycemia increases AMPK activity and decreases PP2A activity, thus playing a key role in tau hyperphosphorylation in the hippocampus of insulin-deficient DM. Although the FBG level achieved euglycemia, a diet control diet and/or combination with AMPK-activated oral anti-diabetic agent treatment should be considered carefully in insulin-deficient DM patients.

\section{Materials and Methods}

\subsection{Animals}

Eleven-week-old, specific pathogen-free male Sprague-Dawley rats purchased from OrientBio (Sungnam, Korea) were used. Upon arrival, the rats were weighed and housed individually. For the measurement of daily food intake, the rats were housed individually until the end of the study. Our study consisted of 2 weeks of adaptation, 5 weeks of induction of diabetes after surgery, and 6 weeks of the diet control period. At 13 weeks of age after 2 weeks of adaptation, the rats were divided into two groups as follows: 5 for the sham operation as a control group (C) and 11 for the pancreatectomy (Px) group. At 18 weeks of age after 5 weeks of induction of diabetes after surgery, the Px group was divided into two groups as follows: 6 for the ad libitum fed group (AL) and 5 for the calorie restricted group (R). The rats were fed standard chow ad libitum based on AIN-76A (Dyets Inc., Bethlehem, PA, USA) and had free access to tap water during the study. Throughout the whole study, all rats were fed $18 \mathrm{kcal} \%$ normal protein chow, and the AL group was fed ad libitum during the whole study, whereas the R group was fed the same rate of daily food intake $(\mathrm{g} / \mathrm{kg}$ of body weight/day) as the $\mathrm{C}$ group. All rats were kept under conditions maintaining a $12 \mathrm{~h}$ light-dark cycle (light on 08:00-20:00 h), temperature $\left(20-23^{\circ} \mathrm{C}\right)$ and relative humidity $(40-65 \%)$. On the last day of the study, rats were anesthetized by $\mathrm{CO}_{2}$ gas after overnight fasting, weighed immediately, and humanely sacrificed. All 
animal protocols were approved by the Institutional Animal Care and Use Committee at Konkuk University (KU10075, Approval date: 2011.01.13.).

\subsection{Partial pancreatectomy}

To generate an insulin-deficient diabetes model in adult rats, we performed a subtotal pancreatectomy at 13 weeks of age. Briefly, we opened the abdominal wall under $0.7 \mathrm{mg} / \mathrm{kg}$ body weight of Zoletil 50 (Virbac, Carros, France) and $0.2 \mathrm{mg} / \mathrm{kg}$ body weight of Rompun (Bayer Korea, Ansan, Korea) anesthesia and removed the pancreatic tissue carefully with a cotton tipped applicator from the spleen to $1 \mathrm{~mm}$ away from the common bile duct without vascular injury. After the surgery, rats were covered with sheets and were incubated under an infrared light to maintain the body temperature in the normal range. Control rats underwent a sham operation without the removal of pancreatic tissue.

\subsection{Food intake, fasting blood glucose (FBG), and body weight}

Food intake (g) was individually measured every day, and an average daily food intake (g/day) was calculated weekly. FBG levels (mg/dl) were measured at 9 am every other week after overnight fasting from tail vein blood using a portable glucometer (Caresense II, Gentrol Co., Incheon, Korea). Body weight was measured every week and on the last day of the study just before they were sacrificed. Every week, daily food intake rates ( $\mathrm{g} / \mathrm{kg} /$ day) of individual rats were determined using an average daily food intake (g/day) and body weight $(\mathrm{kg})$ from a week age.

\subsection{Plasma insulin and C-peptide analysis}

Overnight fasting blood samples were taken from the inferior vena cava for determination of insulin and C-peptide concentrations immediately before the excision of organs. Plasma insulin and C-peptide levels were analyzed using radioimmunoassay kits (Millipore, Billerica, MA, USA) according to the manufacturer's instructions, and the resulting radioactivities were measured using a $\gamma$-counter (Beckman Coulter, Brea, CA, USA).

\subsection{Processing of brain tissue}

At the end of the study, brain tissue was excised from $\mathrm{CO}_{2}$-anesthetized rats and weighed immediately. Both sides of the hippocampus were rapidly separated and flash-frozen in liquid nitrogen for Western blotting. The frozen samples were ground to powder in liquid nitrogen and stored at $-70^{\circ} \mathrm{C}$ until use.

\subsection{Western blot analysis}

The frozen-ground hippocampal tissue samples were homogenized in ice-cold buffer containing $25 \mathrm{mM}$ HEPES, $25 \mathrm{mM}$ benzamidine, $100 \mathrm{mM}$ sodium fluoride, $10 \mathrm{mM}$ sodium pyrophosphate, $2 \mathrm{mM}$ sodium orthovanadate, $1 \%$ Triton X-100, $4 \mathrm{mM}$ EDTA, $5 \mu \mathrm{l} / \mathrm{mL}$ of phosphatase inhibitor cocktail I, 5 $\mu \mathrm{l} / \mathrm{mL}$ of phosphatase inhibitor cocktail II, and $5 \mu \mathrm{l} / \mathrm{mL}$ of protease inhibitor cocktail. After centrifugation at $18,400 \times \mathrm{g}$ for $30 \mathrm{~min}$ at $4^{\circ} \mathrm{C}$, the supernatants were collected, and the protein concentrations were measured by using a BCA kit (Pierce, Rockford, IL, USA) according to the manufacturer's instructions. The extracted proteins were separated on SDS polyacrylamide gels, i.e., $8 \%$ gels for AMPK, PP2A, tau and Akt and 15\% gels for GSK-3 $\beta$. The separated proteins were transferred to nitrocellulose (Bio-Rad Laboratories, Inc., Hercules, CA, USA) membranes at $250 \mathrm{~mA}$ for 1 hour and $30 \mathrm{~min}$. The membranes were blocked by incubating them with $5 \%$ bovine serum albumin buffer for 1 hour at room temperature, and they were then incubated overnight with phospho-Thr ${ }^{172}$ AMPK (1:5,000; Cell Signaling Technology, Inc., Danvers, MA, USA), phospho-PP2A (1:1,000; Millipore, Bellerica, MA, USA), phospho-Ser ${ }^{199 / 202}$ tau (1:1,000; Invitrogen, Camarillo, CA, USA), phospho-Ser ${ }^{396}$ tau (1:1,000; Invitrogen), phospho-Ser ${ }^{473}$ Akt (1:5,000; CST, Inc.), or phosphoSer $^{9}$ GSK-3 $\beta$ antibodies (1:1,000; CST, Inc.) at $4^{\circ} \mathrm{C}$. We also detected total AMPK (1:5,000, CST, Inc.), total PP2A (1:1,000; Millipore), total tau (1:1,000; Invitrogen), total Akt antibody (1:5,000; CST, Inc.), 
or total GSK-3 $\beta$ antibody (1:1,000; CST, Inc.) activity. The membranes were then developed using horseradish peroxidase-conjugated anti-rabbit IgG (1:5000; CST, Inc.) followed by detection with enhanced chemiluminescence (GE Healthcare, Wauwatosa, WI, USA). The immunoreactive protein bands were quantified by using Multi Gague version 3.1 (Fujifilm, Tokyo, Japan).

\subsection{Histology}

Overnight fasted hippocampal tissues were excised and pre-fixed in $4 \%$ paraformaldehyde, embedded in paraffin, and sliced into $5 \mu \mathrm{m}$ thick sections. The hippocampal sections were stained with crystal violet (CV).

\subsection{Immunohistochemistry}

Briefly, the sections were deparaffinized, rehydrated and washed in PBS-TW, treated with 55\% formic acid and $2 \%$ hydrogen peroxide, blocked with $10 \% \mathrm{FBS}$ and incubated overnight at $4^{\circ} \mathrm{C}$ with phospho-Tau (1:500, Invitrogen). Then, the sections were sequentially incubated with peroxidaseconjugated anti-rabbit IgG (1:500; CST, Inc.). Peroxidase activity was developed with $0.05 \% 3,3^{\prime}-$ diaminobenzidine (Sigma) and $0.01 \%$ hydrogen peroxide until the reaction products were visualized (brown color).

\subsection{Statistical analysis}

Data are presented as the mean \pm SD. Statistical analysis was performed using SPSS 18.0 software (SPSS Inc., Chicago, IL, USA). Statistical significance was evaluated using one-way ANOVA with Tukey's post hoc test. $P$ values of less than 0.05 were considered statistically significant.

Author Contributions: Designed the research and wrote the paper: S.H., Y.N. and J.L.; performed the experiments: J.L., M.J. and J.K.; analyzed the data: Y.N., J.L., M.J. and J.K.

Funding: This research received no external funding.

Acknowledgments: This work was supported by Konkuk University in 2014.

Conflicts of Interest: The Authors have no conflicts of interest to declare.

\section{References}

1. Grundke-Iqbal, I.; Iqbal, K.; Tung, Y.C.; Quinlan, M.; Wisniewski, H.M.; Binder, L.I. Abnormal phosphorylation of the microtubule-associated protein tau (tau) in Alzheimer cytoskeletal pathology. Proc. Natl Acad. Sci. USA 1986, 83, 4913-4917. DOI 10.1073/pnas.83.13.4913.

2. Hartmann, T.; Bieger, S.C.; Brühl, B.; Tienari, P.J.; Ida, N.; Allsop, D.; Roberts, G.W.; Masters, C.L.; Dotti, C.G.; Unsicker, K.; et al. Distinct sites of intracellular production for Alzheimer's disease a beta40/42 amyloid peptides. Nat. Med. 1997, 3, 1016-1020. DOI 10.1038/nm0997-1016.

3. Spires-Jones, T.L.; Stoothoff, W.H.; de Calignon, A.; Jones, P.B.; Hyman, B.T. Tau pathophysiology in neurodegeneration: A tangled issue. Trends Neurosci. 2009, 32, 150-159. DOI 10.1016/j.tins.2008.11.007.

4. Maher, P.A.; Schubert, D.R. Metabolic links between diabetes and Alzheimer's disease. Expert Rev. Neurother. 2009, 9, 617-630. DOI 10.1586/ern.09.18.

5. Biessels, G.J.; Staekenborg, S.; Brunner, E.; Brayne, C.; Scheltens, P. Risk of dementia in diabetes mellitus: A systematic review. Lancet. Neurol. 2006, 5, 64-74. DOI 10.1016/S1474-4422(05)70284-2.

6. Arvanitakis, Z.; Wilson, R.S.; Bienias, J.L.; Evans, D.A.; Bennett, D.A. Diabetes mellitus and risk of Alzheimer disease and decline in cognitive function. Arch. Neurol. 2004, 61, 661-666. DOI 10.1001/archneur.61.5.661.

7. Jolivalt, C.G.; Lee, C.A.; Beiswenger, K.K.; Smith, J.L.; Orlov, M.; Torrance, M.A.; Masliah, E. Defective insulin signaling pathway and increased glycogen synthase kinase-3 activity in the brain of diabetic mice: Parallels with Alzheimer's disease and correction by insulin. J. Neurosci. Res. 2008, 86, 3265-3274. DOI 10.1002/jnr.21787. 
8. Qu, Z.; Jiao, Z.; Sun, X.; Zhao, Y.; Ren, J.; Xu, G. Effects of streptozotocin-induced diabetes on tau phosphorylation in the rat brain. Brain Res. 2011, 1383, 300-306. DOI 10.1016/j.brainres.2011.01.084.

9. Stratton, I.M.; Adler, A.I.; Neil, H.A.; Matthews, D.R.; Manley, S.E.; Cull, C.A.; Hadden, D.; Turner, R.C.; Holman, R.R. Association of glycaemia with macrovascular and microvascular complications of type 2 diabetes (ukpds 35): Prospective observational study. BMJ 2000, 321, 405-412. DOI 10.1136/bmj.321.7258.405.

10. Currie, C.J.; Peters, J.R.; Tynan, A.; Evans, M.; Heine, R.J.; Bracco, O.L.; Zagar, T.; Poole, C.D. Survival as a function of $\mathrm{hba}(1 \mathrm{c})$ in people with type 2 diabetes: A retrospective cohort study. Lancet 2010, 375, 481-489. DOI 10.1016/S0140-6736(09)61969-3.

11. MacLeod, J.; Franz, M.J.; Handu, D.; Gradwell, E.; Brown, C.; Evert, A.; Reppert, A.; Robinson, M. Academy of nutrition and dietetics nutrition practice guideline for type 1 and type 2 diabetes in adults: Nutrition intervention evidence reviews and recommendations. J. Acad. Nutr. Diet. 2017, 117, 1637-1658. DOI 10.1016/j.jand.2017.03.023.

12. American Diabetes Association. 4. Lifestyle management: Standards of medical care in diabetes-2018. Diabetes Care 2018, 41, S38-SS50. DOI 10.2337/dc18-S004.

13. Lee, J.H.; Lee, J.H.; Jin, M.; Han, S.D.; Chon, G.R.; Kim, I.H.; Kim, S.; Kim, S.Y.; Choi, S.B.; Noh, Y.H. Diet control to achieve euglycemia induces significant loss of heart and liver weight via increased autophagy compared with ad libitum diet in diabetic rats. Exp. Mol. Med. 2014, 46, e111. DOI 10.1038/emm.2014.52.

14. Lee, J.H.; Choi, S.B.; Jin, M.; Lee, J.H.; Han, S.D.; Bae, H.; Lim, I.; Noh, Y.H. Euglycemia in diabetic rats leads to reduced liver weight via increased autophagy and apoptosis through increased ampk and caspase-3 and decreased mtor activities. J. Diabetes Res. 2015, 2015, 497431. DOI 10.1155/2015/497431.

15. Thornton, C.; Bright, N.J.; Sastre, M.; Muckett, P.J.; Carling, D. Amp-activated protein kinase (ampk) is a tau kinase, activated in response to amyloid beta-peptide exposure. Biochem. J. 2011, 434, 503-512. DOI 10.1042/BJ20101485.

16. Vingtdeux, V.; Davies, P.; Dickson, D.W.; Marambaud, P. Ampk is abnormally activated in tangle- and pre-tangle-bearing neurons in Alzheimer's disease and other tauopathies. Acta neuropathol. 2011, 121, 337-349. DOI 10.1007/s00401-010-0759-x.

17. Desikan, R.S.; Cabral, H.J.; Hess, C.P.; Dillon, W.P.; Glastonbury, C.M.; Weiner, M.W.; Schmansky, N.J.; Greve, D.N.; Salat, D.H.; Buckner, R.L.; et al. Automated MRI measures identify individuals with mild cognitive impairment and Alzheimer's disease. Brain 2009, 132, 2048-2057. DOI 10.1093/brain/awp123.

18. Davies, S.P.; Helps, N.R.; Cohen, P.T.; Hardie, D.G. 5'-amp inhibits dephosphorylation, as well as promoting phosphorylation, of the amp-activated protein kinase. Studies using bacterially expressed human protein phosphatase-2c alpha and native bovine protein phosphatase-2ac. FEBS Lett. 1995, 377, 421-425. DOI 10.1016/0014-5793(95)01368-7.

19. Sontag, J.M.; Sontag, E. Protein phosphatase 2A dysfunction in Alzheimer's disease. Front. Mol. Neurosci. 2014, 7, 16. DOI 10.3389/fnmol.2014.00016.

20. Jeon, S.M. Regulation and function of ampk in physiology and diseases. Exp. Mol. Med. 2016, 48 , e245. DOI 10.1038/emm.2016.81.

21. Ronnett, G.V.; Ramamurthy, S.; Kleman, A.M.; Landree, L.E.; Aja, S. Ampk in the brain: Its roles in energy balance and neuroprotection. J. Neurochem. 2009, 109 (Suppl. 1), 17-23. DOI 10.1111/j.14714159.2009.05916.x.

22. Salminen, A.; Kaarniranta, K.; Haapasalo, A.; Soininen, H.; Hiltunen, M. Amp-activated protein kinase: A potential player in Alzheimer's disease. J. Neurochem. 2011, 118, 460-474. DOI 10.1111/j.14714159.2011.07331.x.

23. Cai, Z.; Yan, L.J.; Li, K.; Quazi, S.H.; Zhao, B. Roles of amp-activated protein kinase in Alzheimer's disease. Neuromolecular Med. 2012, 14, 1-14. DOI 10.1007/s12017-012-8173-2.

24. Clodfelder-Miller, B.; De Sarno, P.; Zmijewska, A.A.; Song, L.; Jope, R.S. Physiological and pathological changes in glucose regulate brain Akt and glycogen synthase kinase-3. J. Biol. Chem. 2005, 280, 39723-39731. DOI 10.1074/jbc.M508824200.

25. Janssens, V.; Goris, J. Protein phosphatase 2A: A highly regulated family of serine/threonine phosphatases implicated in cell growth and signalling. Biochem. J. 2001, 353, 417-439. DOI 10.1042/bj3530417.

26. Tian, Q.; Wang, J. Role of serine/threonine protein phosphatase in Alzheimer's disease. NeuroSignals 2002, 11, 262-269. DOI 10.1159/000067425. 
27. Zhou, X.W.; Winblad, B.; Guan, Z.; Pei, J.J. Interactions between glycogen synthase kinase 3beta, protein kinase $\mathrm{B}$, and protein phosphatase $2 \mathrm{~A}$ in tau phosphorylation in mouse n2a neuroblastoma cells. $J$. Alzheimers Dis. 2009, 17, 929-937. DOI 10.3233/JAD-2009-1113.

28. Clodfelder-Miller, B.J.; Zmijewska, A.A.; Johnson, G.V.; Jope, R.S. Tau is hyperphosphorylated at multiple sites in mouse brain in vivo after streptozotocin-induced insulin deficiency. Diabetes 2006, 55, 3320-3325. DOI 10.2337/db06-0485.

29. Kickstein, E.; Krauss, S.; Thornhill, P.; Rutschow, D.; Zeller, R.; Sharkey, J.; Williamson, R.; Fuchs, M.; Köhler, A.; Glossmann, H.; et al. Biguanide metformin acts on tau phosphorylation via mtor/protein phosphatase 2A (pp2a) signaling. Proc. Natl Acad. Sci. USA. 2010, 107, 21830-21835. DOI 10.1073/pnas.0912793107. 